\title{
Reply to: The many faces of TUBB4A mutations
}

\author{
Lubov Blumkin • Ayelet Halevy • Dominique Ben-Ami-Raichman • Dvir Dahari • \\ Ami Haviv • Cohen Sarit • Dorit Lev • Marjo S. van der Knaap • \\ Tally Lerman-Sagie • Esther Leshinsky-Silver
}

Received: 10 March 2014 / Accepted: 11 March 2014 / Published online: 23 March 2014

(C) Springer-Verlag Berlin Heidelberg 2014

\section{Dear Sirs,}

We have read with interest the letter by Lohmann and Klein regarding our paper Expansion of the spectrum of TUBB4Arelated disorders: a new phenotype associated with a novel mutation in the TUBB4A gene.

Lohmann and Klein expand our knowledge regarding the first family described with DYT4 dystonia in 1985 and agree with us that the more recently described phenotype of

Lubov Blumkin and Ayelet Halevy contributed equally to this work.

L. Blumkin · T. Lerman-Sagie

Pediatric Neurology Unit, Wolfson Medical Center, Holon, Israel

L. Blumkin • D. Lev $\cdot$ T. Lerman-Sagie $\cdot$ E. Leshinsky-Silver Metabolic-Neurogenetic Clinic, Wolfson Medical Center, Holon, Israel

\section{A. Halevy}

Pediatric Neurology and Development Center, Schneider Children's Medical Center of Israel, Petach Tikva, Israel

D. Ben-Ami-Raichman

Diagnostic Imaging Department, Neuroradiology Unit, Wolfson

Medical Center, Holon, Israel

D. Dahari · A. Haviv

Toldot Genetics Ltd, Tel Aviv, Israel

D. Lev

Institute of Medical Genetics, Wolfson Medical Center, Holon, Israel

M. S. van der Knaap

Department of Child Neurology, VU University Medical Center, Amsterdam, The Netherlands

D. Ben-Ami-Raichman • C. Sarit • E. Leshinsky-Silver $(\triangle)$ Molecular Genetics Laboratory, Wolfson Medical Center, Holon, Israel

e-mail: leshinsky@wolfson.health.gov.il

L. Blumkin • D. Lev $\cdot$ T. Lerman-Sagie $\cdot$ E. Leshinsky-Silver Sackler School of Medicine, Tel-Aviv University, Tel-Aviv, Israel hypomyelination with atrophy of the basal ganglia and cerebellum $(\mathrm{H}-\mathrm{ABC})$ is a different and much more severe phenotype.

We described a novel mutation (E410K) in the TUBB4A gene associated with a new phenotype, different from DYT4 dystonia and H-ABC. Lohmann and Klein suggested that the described clinicoradiological phenotype was in keeping with the $\mathrm{H}-\mathrm{ABC}$ spectrum. However, as described in our original paper, our patient demonstrates only mild and slowly progressive leg spasticity with retained ambulation in contrast to the typical presentation of $\mathrm{H}-\mathrm{ABC}$, which shows an earlier and faster progression resulting in a disabling motor disorder. The brain MRI findings are also different, demonstrating only a regional and mild myelin deficit compared to diffuse hypomylination in $\mathrm{H}-\mathrm{ABC}$ and no atrophy of the basal ganglia.

Lohmann and Klein inquired regarding the frequency of the $\mathrm{E} 410 \mathrm{~K}$ variant in ethnically matched controls. This variant is not mentioned in the Exome variant server as well as in the dbSNP databases. No information is available regarding the frequency in Moroccan Jewish population.

Lohmann and Klein mention the fact that there were 29 de novo variants in the patient's DNA, which may have contributed to the phenotype. We have analyzed these genes and found that none of them have been described in association with neurologic symptoms except for IQSEC2. Mutations in this gene have been associated with nonsyndromic intellectual disability and epilepsy, but the variant found in our patient (A1311T) is predicted to be benign according to SIFT, Polyphen2, and mutation taster.

We greatly appreciate the clarification by Lohmann and Klein regarding the confusion in the literature and OMIM nomenclature of the beta tubulin genes. They indicate that the article by Breuss et al. does not deal with mutations in TUBB4A but rather in TUBB5. The table they have added is extremely helpful. We have requested publication of an ERRATUM regarding our paper stating that the paper by Breuss et al. should be omitted from the discussion, table, and reference list, since it does not describe another presentation of TUBB4A 